\title{
Guest Editors' Foreword
}

\author{
Nikhil Bansal $^{1}$. Irene Finocchi ${ }^{2}$
}

Received: 29 March 2017 / Accepted: 1 April 2017 / Published online: 24 April 2017

(C) Springer Science+Business Media New York 2017

We are pleased to present this special issue of Algorithmica, which contains the extended journal versions of selected papers previously presented at the 23rd European Symposium on Algorithms (ESA 2015), held in Patras, Greece, September 14-16, 2015.

ESA is one of the premier conferences on algorithms and data structures. The symposium covers research in all aspects of the design, analysis, engineering, and application of algorithms and data structures. Ever since 2002, it has had two tracks: the Design and Analysis Track (Track A), intended for papers on the design and mathematical analysis of algorithms, and the Engineering and Applications Track (Track B), for submissions dealing with real-world applications, engineering, and experimental analysis of algorithms.

The symposium program was very strong: out of a record number of 320 submissions, the two committees selected 86 papers for inclusion in the scientific program. From that program we have selected 8 outstanding papers for presentation in this special issue, reflecting the diversity of the field of algorithmics. The papers were revised and carefully reviewed according to the high standards of Algorithmica.

In "An Experimental Evaluation of the Best-of-Many Christofides' Algorithm for the Traveling Salesman Problem", Genova and Williamson perform a thorough experimental analysis of recent approximation algorithms for TSP and show that variants based on sampling a spanning tree from an LP relaxation, perform significantly better than Christofides' algorithm. In 'Improved Analysis of Complete-Linkage Clustering”, Großwendt and Röglin give the first constant approximation for metrics induced

\footnotetext{
$\bowtie \quad$ Irene Finocchi finocchi@di.uniroma1.it

1 Eindhoven University of Technology, Eindhoven, The Netherlands

2 Sapienza University of Rome, Rome, Italy
} 
by norms in $O(1)$ dimensions for the complete-linkage clustering algorithm, a popular method for computing hierarchical clusterings. In "How to Sort by Walking and Swapping on Paths and Trees", Graf considers a natural variant of sorting on a tree, motivated by robotic applications and gives an efficient quadratic time algorithm for it. "On the Approximability of Digraph Ordering", by Kenkre et al, presents several approximation for ordering problems. For some cases, it also gives matching integrality gaps for LPs based on several rounds of the Sherali-Adams hierarchy. In "Multicuts in Planar and Bounded-Genus Graphs with Bounded Number of Terminals", de Verdiére presents an FPT algorithm based on topological techniques, for the minimum multicut problem on bounded-genus graphs. "Explicit Expanding Expanders", by Dinitz et al, presents an explicit construction of an infinite family of $d$-regular expanders such that the graph $G_{i}$ has $i$ vertices, and $G_{i}$ and $G_{i+1}$ differ by at most $5 d / 2$ edges. In "Scheduling with Deadlines and Buffer Management with Processing Requirements", Azar and Gilon give substantially improved and tight lower and upper bounds for various classic online problems in scheduling, using simple and elegant proofs. "I/O-Efficient Similarity Join", by Pagh et al, presents an I/O-efficient algorithm for computing similarity joins based on locality-sensitive hashing (LSH), giving a simple, recursive, cache-oblivious procedure with provable sub-quadratic dependency on the data size.

We are grateful to the authors and to the referees for their hard work in bringing this special issue to fruition. Many of these papers are substantial improvements over the already-excellent versions presented at the symposium, and this is due to the diligence, commitment, and energy of the authors and the referees. We are also grateful to the Editor-in-Chief, Ming-Yang Kao, for his guidance and assistance. We hope the readers will enjoy the contents of the present issue.

Nikhil Bansal and Irene Finocchi Program Co-Chairs of the 23rd European Symposium on Algorithms 